\title{
Fuzzy Tuned Gossip Algorithms in Mobile Ad Hoc Networks
}

\author{
Athanasios N. Tsapanoglou, Student Member, IEEE and Dimitrios P. Iracleous, Member, IEEE
}

\begin{abstract}
Mobile ad hoc networks (MANET) are modeled as agents that form communities without infrastructure, for a random period of time and with usually cooperative behavior. The nodes of MANET often carry information to disseminate. The dynamics of information delivery, mostly referred as average consensus, is a common problem in these networks. The gossip protocols are designed to implement this task. The standard algorithms that are used in these protocols exploit the network describing matrix, aka Laplacian, and exchange information to all node neighbors. In static networks the problem can be considered as an output feedback problem but in the case of MANET the problem is getting complicated due to the continuous change of network topology. In this paper the fuzzy reasoning approach is proposed to tune and leverage the gossip protocol. Illustrative simulations are included to demonstrate the application of the method and to present comparative results in various cases.
\end{abstract}

Index Terms-Average Consensus Problem, Fuzzy Tuning, Gossip Protocol, Mobile Ad Hoc Networks.

\section{INTRODUCTION}

OSSIP algorithms were initially introduced by Tsitsiklis [1], in 1984, to achieve consensus over a set of agents. They are implemented in gossip protocols, also known as epidemic or distributed algorithms, and intend to find a way of agreement about local information that are regarded and communicated as real numbers [2], [3]. The protocols are distributed messagepassing schemes designed to broadcast and process information over multi agent systems, MANET or swarms [4]. Since they constitute simple and robust methods for distributed information processing, they have recently received significant attention from researchers [5]-[7].

MANET are wireless networks characterized by the absence of a fixed infrastructure. They are decentralized and they do not have any components in order to support individually any specific network functionality. Although MANET were initially used in fields like military and recovery operations, they have permeated into our daily life in common devices, including laptops, sensors, PDAs and mobile phones, through technologies that enable ad hoc networking, like Bluetooth and IEEE 802.11b [8]-[9].

Manuscript received January 31, 2009

A.N. Tsapanoglou is IST Studies/ University of Hertfordshire, 72 Pireos Str., 183 46, Moschato, Athens, Greece (phone: (+30) 2104822-222, fax. (+30) 210-4821-850; e-mail: tsapanoglou@ ieee.org).

D. P. Iracleous is with the IST Studies/ University of Hertfordshire, 72 Pireos Str., 183 46, Moschato, Athens, Greece (phone: (+30) 2104822-222, fax. (+30) 210-4821-850; e-mail: iracleous@ ieee.org).
Several control approaches have been proposed to enhance the consensus behavior of MANET, sensor networks or multi agent systems [10]-[13].

This paper examines the fuzzy logic in order to introduce a more effective way of broadcasting information in MANET using gossip algorithms, as was used in routing [14]. The method, which is a biological inspired algorithm, is an approach of using knowledge in a way similar to human brain.

In the following a formal analysis of the network presentation is given and the dynamics of the consensus problem is derived. The fuzzy algorithm is illustrated and simulation results depict the performance of the fuzzy tuned protocol.

\section{THEORETICAL BACKGROUND}

\section{A. MANET system description}

MANET are characterized by continuous change of the network topology. The network can be described as a set of nodes and edges. At every instant a Laplacian matrix defined to identify the possible interconnection between the nodes [6].The Laplacian is a symmetric $\mathrm{NxN}$ matrix, where $\mathrm{N}$ is the number of the nodes in the MANET and is given as

$$
L_{i j}=\left\{\begin{array}{ll}
-d_{i j} & i \neq j \\
\sum_{i=1}^{N} d_{i j} & i=j
\end{array}, i, j \in[1 . . N]\right.
$$

where $d_{i j}$ is the metric between the nodes i, j. In MANET if two nodes are communicating $d_{i j}$ is set to 1 and if they are not, it is set to 0 .

If all nodes are communicating the networks is called fully connected or full mesh. Fully connected network is a rather costly configuration and partial connected is the usual case. In partial connected networks nodes can communicate either directly or indirectly via another node. In the case that two nodes cannot communicate directly or indirectly the network is called isolated and can considered that consists of two or more independent areas that information flow is impossible.

In MANET, each node has its own clock which ticks independently as a rate $\theta$ Poisson process. Nevertheless, the network's state changes across time, therefore an aggregated synchronous time model is used [15]. In the following scenario a global clock is used which effectively contributes in the proposed algorithm's operation, due to the distributed nature of ad hoc networks. As a result, the inter-tick times are exponentially distributed, and independent across nodes 
and across time.

\section{B. Consensus problem and gossip protocol}

Each node is considered with local knowledge stored as a real number. This information $\mathrm{x}_{\mathrm{i}}(\mathrm{k})$ is the state of $\mathrm{i}^{\text {th }}$ node $i \in[1,2, \ldots, N]$ at every instant $\mathrm{k}$, where $k \in[1,2, \ldots, \infty]$ time instants given by the global clock and can be communicated to neighboring nodes, as shown in the Laplacian matrix.

Definition 1. A gossip protocol is the definition of the messages that are sent from and to the nodes of a network to disseminate information.

The $\mathrm{N}$ nodes have an initial information at time $\mathrm{k}=0$, which represent the total information in the network. The total information, or the average initial state, is given by

$$
\bar{x}=\frac{1}{N} \sum_{i=1}^{N} x_{i}(0) \text {. }
$$

Definition 2. An average consensus algorithm is the suitable algorithm that defines the necessary messages between the nodes in other to achieve the as final state $\bar{x}$ to all nodes.

Average consensus can be accomplished after a finite number of steps or asymptotically, i.e.

$$
\lim _{k \rightarrow \infty} x_{i}(k)=\bar{x} \quad \forall i \in[1,2, . . N] \text {. }
$$

A proposal for a consensus algorithm to be implemented in a gossip protocol has in general the following dynamics

$$
x_{i}(k+1)=f\left(x_{i}(k)\right)+g\left(x_{j}(k)\right)
$$

where $\mathrm{k}$ is the global clock, and $f(\cdot)$ and $g(\cdot)$ suitable functions that implement feedback.

If we collect all node states to the network information vector as

$$
x=\left[\begin{array}{c}
x_{1} \\
x_{2} \\
\cdots \\
x_{N}
\end{array}\right]
$$

and supposing a linear model for functions $f(\cdot)$ and $g(\cdot)$ we can derive the following linear model for the consensus problem

$$
x(k+1)=A(k) x(k)
$$

Using (6), it is derived that

$$
\lim _{k \rightarrow \infty} x(k)=A(\infty) \cdots A(1) A(0) x(0)
$$

Using (3) and (7) we derive that the gossip protocol for average consensus is the suitable definitions of $A(0), A(1), \cdots, A(\infty)$ to satisfy

$$
A(\infty) \cdots A(1) A(0) x(0)=\bar{x} 1
$$

where 1 is a Nx1 vector with all its elements 1 .

A gossip protocol will be given as the series of matrices $A(\cdot)$, i.e.

$$
A(k)=\aleph(k)
$$

to achieve (8).

In a static network, i.e

$$
A(0)=A(1)=\cdots=A(\infty)=A
$$

the problem is reduced to an output feedback problem. A simple solution to the problem is given as

$$
A=\left(I-\frac{1}{N} L\right)
$$

where I is the $\mathrm{NxN}$ identity matrix [16].

In MANET, the system matrix is varying. An analogous solution can be given according to a suitable mobility model of the corresponding nodes [17]. Also certain constraints should be taken into account, due to the nature of the system mobility.

\section{Biological inspired algorithms}

The gossip protocol for the average consensus problem is rather complicated in MANET. Detailed formal algorithms require detailed model of the controlled systems and explicit bounds of the tolerated disturbances. It is similar to generally unsolved output feedback stabilization problem in time varying and uncertain systems.

On the other hand humans deal with uncertainties quite effectively. They use qualitative expressions to describe the state of processes, instead of quantitative variables. Human experts effectively reason without rigor mathematical manipulations. This logic is a biological algorithm, which inspire researchers to create a similar approach. In order to imitate this biological process fuzzy reasoning was proposed by Zadeh. The success of this approach is based on suitable definition of the linguistic domain of the problem and the rules that are derived by the years of experience.

The fuzzy reasoning consists of three basic steps, namely fuzzification, use of rules and defuzzification. These steps constitute the fuzzy tuned algorithm, instead of a formal mathematical formula.

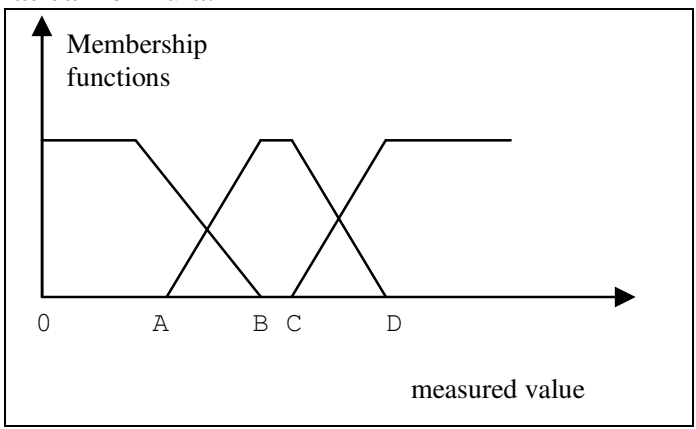

Fig. 1. Membership function used in a standard fuzzification process. A, $\mathrm{B}, \mathrm{C}$ and $\mathrm{D}$ denote the critical values of the membership values.

In the fuzzification step the measurements $a_{i}$ of the monitored process are transformed to linguistic variables $A_{i}$ with the percentage given by the membership functions $\mu(\cdot)$ as shown in Fig. 1 .

The use of the fuzzy rules is the usage of the rules that contain the knowledge base of the system. These rules are 
given by a fuzzy relation $\mathrm{R}$ is assigned by the function $\mu_{R}$ as follows

$\mu_{R}\left(a_{1}, a_{2}, \ldots, a_{N}\right)=\wedge\left(\mu_{A 1}\left(a_{1}\right), \ldots, \mu_{A N}\left(a_{N}\right)\right)$

where $\wedge$ means minimum, $a_{j}$ 's are the input variables and $\mathrm{A}_{j}$ 's are the respective linguistic variables.

The defuzzification step is the final step and uses a mapping from linguistic rules into crisp (numerical) actions. Among to many methods, the Center of Gravity (COG) is selected, defined by the following equation

$$
c v=\frac{\sum_{i=1}^{r} \mu_{R i}\left(a_{1}, \ldots, a_{N}\right) L_{R i}}{\sum_{i=1}^{r} \mu_{R i}\left(a_{1}, \ldots, a_{N}\right)}
$$

where $\mathrm{cv}$ is the crisp value of the controller, $L_{R i}$ is the value of each linguistic variable

\section{PROPOSED ALGORITHM}

In this paper fuzzy reasoning will be used to provide an easy to model and effective approach to define the gossip protocol.

Two linguistic variables will be used, namely vicinity and level of information. The two variables will describe how a node perceives the other nodes. Vicinity of nodes and level of information of each node are described by fuzzy sets. These variables are described in Table I.

TABLE I

LINGUISTIC VARIABLES

\begin{tabular}{ccc}
\hline $\begin{array}{c}\text { Measuring } \\
\text { quantity }\end{array}$ & Linguistic variable & Range \\
\hline Node vicinity & near & $0-1.5$ \\
& intermediate distance & $1-5$ \\
& far & $3-\infty$ \\
\hline Level of & High negative & $-\infty,-5$ \\
information & medium negative & $-7--2$ \\
& Zero or low & $-4-+4$ \\
& medium positive & $+2-+7$ \\
& High positive & $+5-+\infty$
\end{tabular}

The membership functions of each linguistic variable are used by the suitable adaption of Fig. 1 .

Each node can measure the vicinity of the neighboring nodes, as well as their state value. The actual sensor readings are only of limited utility. To take any control action the measurements are transformed to the linguistic variables.

The basic rules base of the fuzzy tuned gossip controller the following guidelines are heuristically proposed [5], [14]:

i) nodes with high vicinity are preferred since their signal is clearer and noise is avoided;

ii) nodes with high negative values are excluded in the communication since they provide fluctuations;

iii) nodes with high positive values are used in the communication since they provide the desired information;

iv) If the neighboring node has the same level of information with the current node is neglected.

Thus, the linguistic variable corresponding to the monitored variable is characterized by fuzzy uncertainty. To emphasize the point, it should be stated that the MANET uncertainty cannot be modeled effectively using probabilistic uncertainty, but requires fuzzy uncertainty.

After the setup of the fuzzy algorithm the gossip protocol $\aleph(k)$ is calculated at every instant using (12) and (13).

\section{SIMULATION STUDIES}

In Fig. 2 a network of 6 nodes is shown at time zero. The MANET is a not fully connected and not isolated network. The system is time varying and but for sake of simplicity it is considered always not isolated.

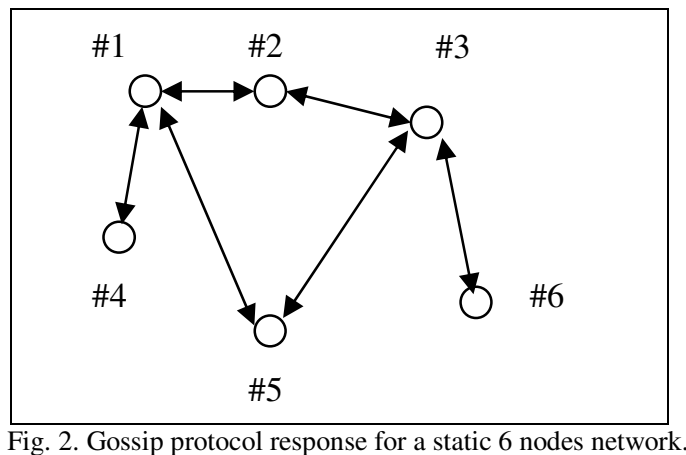

A comparative study of the response of this MANET follows.

Case 1. The system is static and a static gossip protocol is used. The node response is depicted in Fig. 3 where nodes achieve average consensus in about 25 instants.

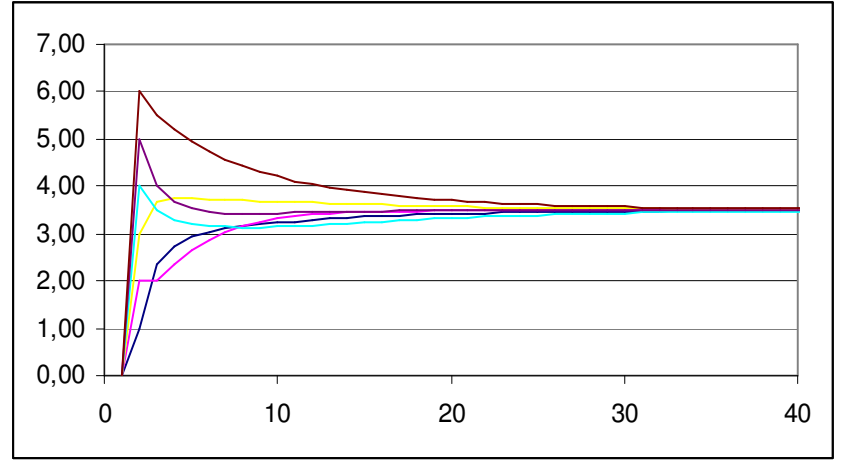

Fig. 3. Gossip protocol response for a static 6 nodes network with constant communicating matrix A.

Case 2. The system is static and a fuzzy tuned gossip protocol is used. Obviously, the fuzzy controller is based only on the level of information of each node and not on their vicinity as it is constant. The node response is depicted in Fig. 4 where nodes achieve average consensus in about 14 instants. 


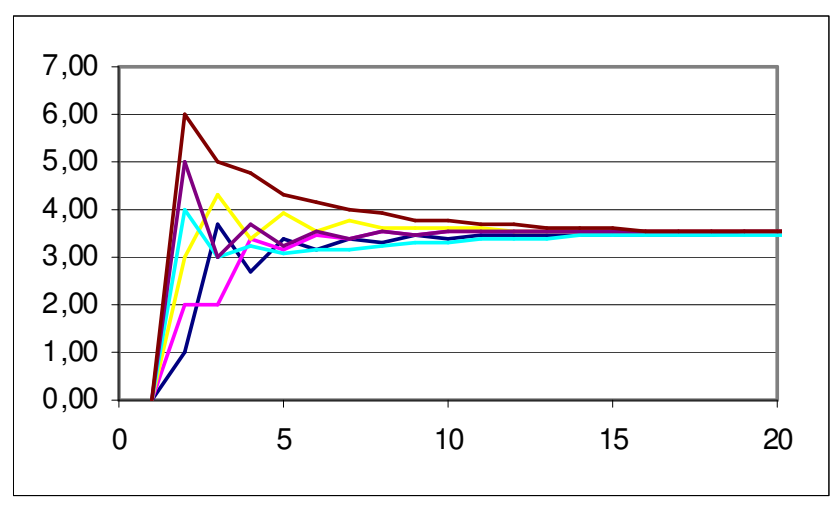

Fig. 4. Gossip protocol response for a static 6 nodes network using the fuzzy tuned communicating matrix A.

Case 3. The system is dynamic moving and a fuzzy tuned gossip protocol is used. The fuzzy controller is based on both the level of information of each node and their vicinity. The node response is depicted in Fig. 5 where nodes achieve average consensus in about 12 instants. Significant fluctuations appear since the nodes are dynamic. In Fig. 6 the evolution of system eigenvalues are depicted.

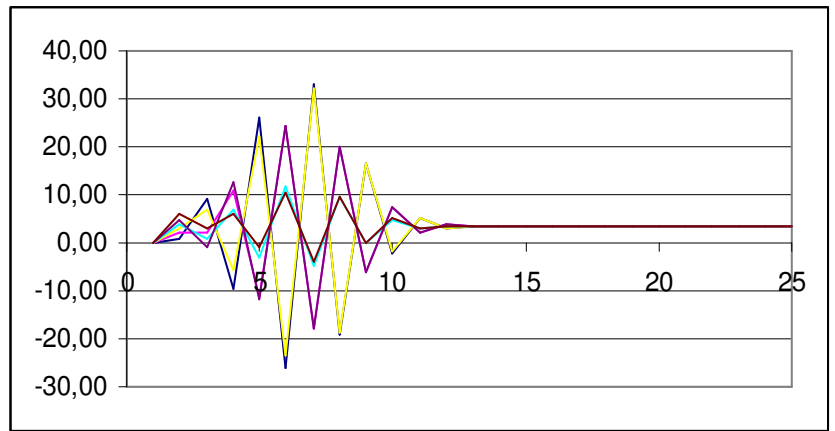

Fig. 5. Gossip protocol response for a dynamic 6 nodes network using the fuzzy tuned communicating matrix A.

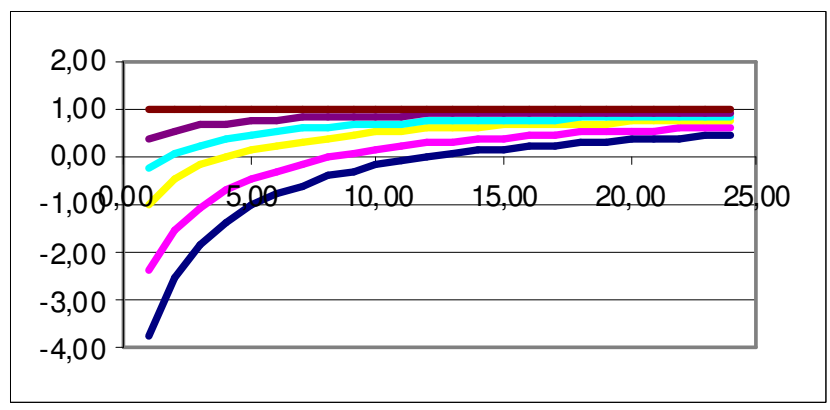

Fig. 6. The eigenvalues of matrix $\mathrm{A}(\mathrm{k})$ during time for the case 3 .

\section{CONCLUSION}

MANETs, regarded as multi agent systems under decentralized guidance, are considered to design a suitable controlling scheme. The time varying model is rather cumbersome for an exact mathematical solution. Therefore, a fuzzy approach is proposed based on the basic rules obtained by the local network discovery of each node. The core advantage of the presented method is that it improves the system performance and can be implemented quite easily.

\section{REFERENCES}

[1] J. Tsitsiklis, "Problems in Decentralized Decision Making and Computation," in Electrical Engineering and Computer Science. vol Doctor of Philosophy: MIT, 1984, p. 271.

[2] P. Yang, R. A. Freeman, K. M. Lynch, "Multi-Agent Coordination by Decentralized Estimation and Control," Automatic Control, IEEE Transactions on, Volume 53, Issue 11, Dec. 2008 Page(s):2480 2496.

[3] B.J. Moore, K.M. Passino, "Distributed Task Assignment for Mobile Agents," Automatic Control, IEEE Transactions on, Volume 52, Issue 4, April 2007 Page(s):749 - 753.

[4] Liu B., et al., "Swarm dynamics of a group of mobile autonomous agents," Chin. Phys. Lett., Vol. 22, No. 1, 2005, pp. 254-257.

[5] L. Tao, Z. Ji-Feng, "Asymptotically Optimal Decentralized Control for Large Population Stochastic Multiagent Systems," Autom. Control, IEEE Trans. On, V. 53, Issue 7, Aug. 2008 Page(s):1643 1660.

[6] S. Kraus, et al, "A Study on Gossiping in Transportation Networks," IEEE Trans. on Vehicular Technology, vol. 57, pp. 2602-2607, 2008.

[7] Y. Shavitt and A. Shay, "Optimal Routing in Gossip Networks," IEEE Trans. on Vehicular Technology, vol. 54, pp. 1473-1487, 2005.

[8] E. Huang, W. Hu, J. Crowcroft, I. Wassell,"Towards Commercial Mobile Ad Hoc Network Applications: A Radio Dispatch System," MobiHoc'05, May 25-27, 2005, Urbana-Champaign, Illinois, USA.

[9] L. Yanfei, K.M. Passino, "Cohesive Behaviors of Multiagent Systems With Information Flow Constraints," Automatic Control, IEEE Transactions on, Volume 51, Issue 11, Nov. 2006 Page(s):1734 1748.

[10] B. Nazer, et al, "Local Interference Can Accelerate Gossip Algorithms," Proceedings of the 46th Annual Allerton Conference on Communication, Control and Computation, Monticello, IL, September 2008.

[11] M. Schmalz, M. Fujita, O. Sawodny, Directed Gossip Algorithms, "Consensus Problems, and Stability Effects of Noise Trading," Computational Methods for Modelling and learning in Social and Human Sciences Bilingual Conference, 10-11 May 2007.

[12] M. Akdere, C.C. Bilgin, O. Gerdaneri, I. Korpeoglu, "A comparison of epidemic algorithms in wireless sensor networks," Computer Communications, 29 (2006) 2450-2457.

[13] L. Mihaylova, D. Angelova, "Algorithms for Mobile Nodes SelfLocalisation in Wireless Ad Hoc Networks," In Proc. of the 9th Intl. Conf. on Information Fusion, 2006.

[14] S. Rea, D. Pesch, "Multi-metric routing decisions for ad hoc networks using fuzzy logic," Wireless Communication Systems, 1 st International Symposium on, Volume , Issue , 20-22 Sept. 2004 Page(s): 403 - 407

[15] B. Sundararaman, U. Buy, A.D. Kshemkalyani, "Clock synchronization for wireless sensor networks: a survey," Ad Hoc Networks, Volume 3, Issue 3, May 2005, Pages 281-323.

[16] D. Kempea, and F. McSherryb, "A decentralized algorithm for spectral analysis," Journal of Computer and System Sciences, Volume 74, Issue 1, February 2008, Pages 70-83.

[17] A.D. Sarwate and A.G. Dimakis, "The Impact of Mobility on Gossip Algorithms," to appear in Infocom 2009.

Athanasios N. Tsapanoglou (SM'07) was born in Athens in 1983. He received the BSc in Computer Studies from University of Sunderland and he is now a postgraduate student at the University of Hertfordshire with the aim of obtaining the degree of MSc (Hons) in Distributed Systems and Networks.

Dimitris P. Iracleous (M'00) was born in Athens in 1969. He received his Diploma in Electrical Engineering from University of Patras in 1993 and his Ph.D. in the area of Automatic Control of Power Systems in 1999 from the Electrical and Computer Engineering Department in the same University.

$\mathrm{He}$ is a Lecturer at IST Studies/ University of Hertfordshire. He is an author of 9 papers in international scientific journals and 19 papers in proceedings of international scientific conferences. He has participated in various research projects funded by the European Union and has been cited by 6 authors in the scientific literature. His research interests include automatic control applications in computer and robotic systems. 